\section{How to best manage cytomegalovirus infection in the allogeneic stem cell transplant setting: future prospects}
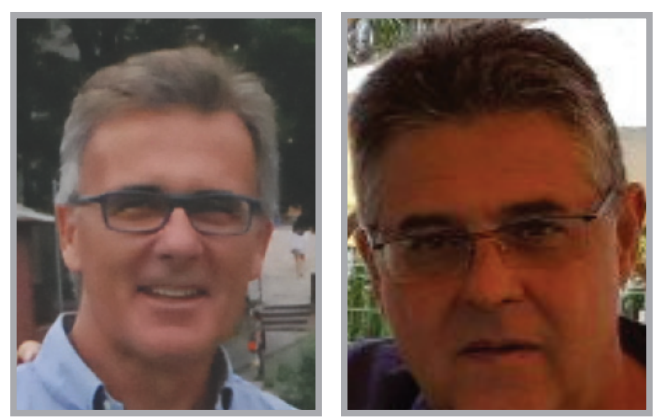

“The use of antivirals with relatively high intrinsic activity against cytomegalovirus ... has dramatically decreased

the incidence of early cytomegalovirus disease...”

Carlos Solano ${ }^{1,2} \&$ David Navarro ${ }^{* 3,4}$

First draft submitted: 9 August 2016; Accepted for publication: 16 August 2016;

Published online: 30 August 2016

Allogeneic stem cell transplantation (alloSCT) was systematically adopted in clinical practice in the early 70 s of the past century, and it soon became the sole curative procedure for a number of hematological malignancies refractory to conventional chemotherapy treatment regimens. In these early days, cytomegalovirus (CMV) infection emerged as a dreadful threat for allo-SCT patients, more than a third of them developing end-organ disease, most frequently pneumonitis or gastrointestinal disease, whose treatment was often unsuccessful [1]. The use of antivirals with relatively high intrinsic activity against CMV, such as (val)ganciclovir or foscarnet, administered either prophylactically or pre-emptively, has dramatically decreased the incidence of early CMV disease (occurring within the first 100 days after transplant), which is currently diagnosed in less than $5 \%$ of allo-SCT recipients [1,2]. Universal prophylaxis, consisting of the administration of antivirals to all patients, usually at the engraftment, irrespective of their particular risk of CMV disease, leads invariably to overtreatment, as many patients who would remain free of CMV disease are nevertheless treated. In addition, development of late-onset disease (occurring after day 100 after transplant), once the antiviral is discontinued, most likely as a result of a suboptimal reconstitution of CMV-specific T-cell immunity, is a non-negligible drawback [1]. Moreover, drug-related toxicity (hematological, most notably) is a major side effect of this approach [1]. Because of all the above, the use of this strategy is restricted to highrisk patients and only applied in very few

\section{KEYWORDS}

cytomegalovirus $\bullet$ pre-emptive antiviral therapy $\bullet$ T-cell immunity - universal prophylaxis $\bullet$ viral doubling time $\bullet$ viremia

"Universal prophylaxis, consisting of the administration of antivirals to all patients, usually at the engraftment, irrespective of their particular risk of cytomegalovirus disease, leads invariably to overtreatment..."

'Hematology Service, Hospital Clínico Universitario, Instituto de Investigación INCLIVA, Valencia, Spain 
"Functional

cytomegalovirus-specific

$T$ cells are essential in the prevention and resolution of viremic episodes." centers. The advent of new antivirals with an increased intrinsic activity against CMV, and a safer toxicity profile, such as maribavir, brincidofovir and specially letermovir, has renewed the interest in universal prophylaxis [1]. Yet these drugs are not totally harmless and as it has been recently shown, viral resistance may emerge during treatments, a potential event that we cannot afford [3]. In this setting, a reasonable goal would be to administer antiviral prophylaxis exclusively to those patients carrying a truly increased risk of viremia (targeted prophylaxis). Unfortunately, pre- and post-transplant clinical factors (i.e., type of transplant, donor/recipent $\mathrm{CMV}$-serostatus, occurrence of severe acute graft vs host disease), known to modulate that risk, are insufficiently predictive. We hypothesized that this approach may be feasible by combining clinical, genetic, biological and immunological data of patients to build a predictive risk score [1] . Genetic traits predisposing to the development of CMV viremia are being increasingly reported. In fact, donor or recipient single nucleotide polymorphisms (SNPs) located within genes (or close to them) regulating innate and adaptive immune responses to CMV, such as Tolllike receptor 2 (TLR-2), chemokine receptor-5 (CCR-5), monocyte chemoattractant protein-1 (MCP-1) or interferon $\lambda 3$ (IL28B) have been shown to impact on the risk and the dynamics of CMV viremia [1]. Large studies proving this assumption are nevertheless lacking. Moreover, sequential measurement of plasma levels of proinflammatory cytokines, prospective metabolomic profiling of plasma specimens and monitoring of $\mathrm{CMV}$-specific $\mathrm{CD} 4^{+}$and $\mathrm{CD} 8^{+} \mathrm{T}$-cell responses by the use of HLA-peptide multimers, flow cytometry for cytokine staining, ELISPOT or by the commercially-available Quantiferon CMV assay were shown to allow anticipation of the occurrence of viremia with reasonable good positive predictive values (see [1] for a review). Naturally, much more work needs to be done in this area, but preliminary data warrants further investigations.

Pre-emptive antiviral therapy, consisting of the inception of antiviral treatment upon detection of viremia is currently the standard of care for the prevention of CMV disease in allo-SCT recipients [1,4]. At most transplant centers, monitoring of CMV DNA load either in plasma or whole blood by real-time quantitative PCR assays (QRT-PCRs) is performed to make decisions as to when to start and when to stop antiviral treatment. The CMV DNA load cutoff prompting the initiation of antiviral therapy varies widely across centers [1-2,4]. Most transplant programs use a pre-established CMV DNA load threshold, irrespective of the patient clinical risk of CMV disease (between 500 and $1500 \mathrm{IU} / \mathrm{ml}$ for plasma or between 1000 and $10,000 \mathrm{IU} / \mathrm{ml}$ for whole blood). Other centers follow a risk-adapted strategy, starting antiviral therapy upon plasma CMV DNA loads as low as $100-150 \mathrm{IU} / \mathrm{ml}$ in high-risk patients [4]. Likewise, antiviral therapy is interrupted upon the first undetectable viral load result in some centers or after two consecutive negative PCR results in others $[1,4]$. There is little doubt that achieving consensus criteria for triggering and interrupting pre-emptive antiviral therapy would be beneficial for patients. This has not been possible because a straight comparison of experiences across centers is not feasible due to the exceeding inter-assay and inter-laboratory coefficients of variation of QRT-PCR assays and the variable efficiency of DNA extraction methods $[5,6]$.

The advent of international standards for CMV DNA load quantitation, in particular the WHO International Standard from the National Institute of Biological Standards and Controls [7], which allows normalization of CMV DNA load values between assays, now reported in $\mathrm{IU} / \mathrm{ml}$, raised great expectations. Nevertheless, the commutability of these standards is suboptimal [8], and as it could be anticipated, significant interassay and inter-center variations for individual specimens persist [9].

The limit of detection of CMV QRT-PCR assays has been lowered dramatically in recent years (20-35 IU/ml, for some of them). In this context, a reasonable question is why withhold antiviral treatment until a given CMV DNA load in blood is reached? A reasonable answer is that by doing so, antiviral treatment can be spared to almost $50 \%$ of viremic patients who would be capable of controlling CMV replication upon a prompt activation and expansion of functional virus-specific $\mathrm{T}$ cells, thus avoiding drug-related toxicity. This strategy assumes that the presence of CMV in the blood compartment is permissible as long as it does not reach a level that would put the patient at an imminent risk of overt CMV disease (>1000-1500 IU/ml). This view, nevertheless, has been recently challenged by Green et al. [10] who reported that CMV DNA loads as low as $250 \mathrm{IU} / \mathrm{ml}$, reached upon the first 
100 days after transplant, are associated with an increased risk of non-relapse death caused by infectious complications, a phenomenon presumably related to the immunosuppresive properties of CMV (indirect effects). These data are difficult to reconcile with earlier findings demonstrating that prophylactic administration of ganciclovir at engraftment and delaying the start of ganciclovir until high-grade antigenemia resulted in similar survival rates [11], and thus must be confirmed in multicenter prospective studies. Likewise, the idea that early initiation of pre-emptive therapy (at viral loads as low as $135 \mathrm{IU} / \mathrm{ml}$ ) may result in shorter courses of antiviral therapy [12] merits further research. Until then, to limit drug overexposure and thus toxicity, the focus must be placed on treating as few patients as possible, without compromising their safety, and using antiviral treatment courses as short as possible, without increasing the patient's risk of relapsing/recurrent episodes and late end-organ disease. We believe that kinetic analyses of plasma CMV DNA load and routine monitoring of CMV-specific T-cell responses may help to achieve these purposes. In effect, we recently showed that a CMV DNA load doubling time $(\mathrm{dt}) \leq 2$ days, anticipated the eventual need of preemptive antiviral with a positive predictive value of $100 \%$ [13]. We later reported that treating patients upon $\mathrm{dt}_{\mathrm{s}} \leq 2$ days led to a significant reduction of the time on antiviral therapy [14]. In addition, we proved [15] that the CMV DNA dt may be a valuable parameter for interassay and inter-institutional results comparison since QRT-PCR assays show linearity above their limit of quantification, with slope coefficients that vary minimally when testing standard calibration panels [9].

Functional CMV-specific T cells are essential in the prevention and resolution of viremic episodes (reviewed in [1]). Sequential monitoring of CMV-specific T-cell responses has been shown to identify those patients of whom antiviral treatment can be spared safely at the time of CMV viremia detection [16], and may help to tailor individually the length of antiviral therapy courses. Previous work by our group showed that patients treated preemptively with antivirals and expanding CMV-specific phosphoprotein (pp) 65 and immediate-early (IE) -1 interferon (IFN) $-\gamma$ $\mathrm{CD}^{+} \mathrm{T}$ cells at levels $>1$ cell $/ \mu \mathrm{l}$ at the time of CMV DNAemia clearance had a reduced risk of developing recurrent episodes of viremia, even using shorter courses of antiviral therapy [17]. We recently conducted a multicenter, closelymatched comparison-group study in which this assumption was found to be correct for a subset of patients [18]. Surely, the near future will witness the resurgence of the debate on whether universal prophylaxis with the new anti-CMV drugs or pre-emptive antiviral therapy must be the standard of care for the prevention of CMV-related clinical complications in allo-SCT recipients. In the meantime, research efforts should be concentrated on identifying precisely which patients are at high risk of developing uncontrolled viremia (tributary of targeted prophylaxis), determining whether low-level viremia has a tangible detrimental effect on patient survival, reducing the inter-assay variability of QRT-PCR assays, evaluating the validity of the $\mathrm{dt}$ for inter-center comparisons, and determining to what extent routine immunological monitoring may contribute to improving the management of CMV infection in these patients by conducting well-designed randomized controlled clinical trials.

\section{Acknowledgements}

The authors are grateful to the residents from the Microbiology Service and the staff from the Hematology Service of Hospital Clinico Universitario, Valencia.

\section{Financial disclosure}

This work was supported by a grant (EC10-317) from the program 'Fomento de la Investigación Clínica Independiente' from Ministerio de Sanidad, Politica Social e Igualdad, Spain and by a grant (FIS 12/01992) from Fondo de Investigaciones Sanitarias (FIS), Ministerio de Sanidad y Consumo, Spain. The authors have no other relevant affiliations or financial involvement with any organization or entity with a financial interest in or financial conflict with the subject matter or materials discussed in the manuscript apart from those disclosed.

No writing assistance was utilized in the production of this manuscript.

\section{References}

1 Boeckh M, Nichols WG, Papanicolaou G, Rubin R, Wingard JR, Zaia J.

Cytomegalovirus in hematopoietic stem cell transplant recipients: current status, known challenges, and future strategies. Biol. Blood Marrow Transplant. 9(9), 543-558 (2003).

2 Solano C, de la Cámara R, Vázquez L, López J, Giménez E, Navarro D. Cytomegalovirus infection management in allogeneic stem cell transplant recipients: a national survey in Spain. J. Clin. Microbiol. 53(8), 2741-2744 (2015).

3 Lischka P, Michel D, Zimmermann H. Characterization of cytomegalovirus 
breakthrough events in a Phase 2 prophylaxis trial of Letermovir (AIC246, MK 8228). J. Infect. Dis. 213(1), 23-30 (2016). Boeckh M, Ljungman P. How we treat cytomegalovirus in hematopoietic cell transplant recipients. Blood 113(23), 5711-5719 (2009).

5 Pang XL, Fox JD, Fenton JM, Miller GG, Caliendo AM, Preiksaitis JK. American Society of Transplantation Infectious Diseases Community of Practice; Canadian Society of Transplantation Interlaboratory comparison of cytomegalovirus viral load assays. Am. J. Transplant. 9(2), 258-268 (2009).

6 Bravo D, Clari MA, Costa E et al. Comparative evaluation of three automated systems for DNA extraction in conjunction with three commercially available real-time PCR assays for quantitation of plasma cytomegalovirus DNAemia in allogeneic stem cell transplant recipients. J. Clin. Microbiol. 49(8), 2899-2904 (2011).

7 Fryer JF, Heath AB, Anderson R, Minor PD, the Collaborative Study Group. Collaborative study to evaluate the proposed 1st who international standard for human cytomegalovirus (HCMV) for nucleic acid amplification (NAT)-based assays (WHO/BS/10.2138) (2010). www.nibsc.org/documents/ifu/09-162.pdf

8 Hayden RT, Preiksaitis J, Tong Y et al. Commutability of the first World Health Organization international standard for human cytomegalovirus. J. Clin. Microbiol. 53(10), 3325-33 (2015).
9 Preiksaitis JK, Hayden RT, Tong Y et al. Are we there yet? Impact of the first international standard for cytomegalovirus DNA on the harmonization of results reported on plasma samples. Clin. Infect. Dis. 63(5), 583-589 (2016).

10 Green ML, Leisenring W, Xie $\mathrm{H}$ et al. Cytomegalovirus viral load and mortality after haemopoietic stem cell transplantation in the era of pre-emptive therapy: a retrospective cohort study. Lancet Haematol. 3, e119-e127 (2016).

11 Boeckh M, Gooley TA, Myerson D, Cunningham T, Schoch G, Bowden RA. Cytomegalovirus pp65 antigenemia-guided early treatment with ganciclovir versus ganciclovir at engraftment after allogeneic marrow transplantation: a randomized double-blind study. Blood 88(10), 4063-4071 (1996).

12 Tan SK, Waggoner JJ, Pinsky BA. Cytomegalovirus load at treatment initiation is predictive of time to resolution of viremia and duration of therapy in hematopoietic cell transplant recipients. J. Clin. Virol. 69, 179-183 (2015).

13 Giménez E, Muñoz-Cobo B, Solano C, Amat P, Navarro D. Early kinetics of plasma cytomegalovirus DNA load in allogeneic stem cell transplant recipients in the era of highly sensitive real-time PCR assays: does it have any clinical value? J. Clin. Microbiol. 52(2), 654-656 (2014).

14 Solano C, Giménez E, Piñana JL et al. Preemptive antiviral therapy for CMV infection in allogeneic stem cell transplant recipients guided by the viral doubling time in the blood. Bone Marrow Transplant. 51(5), 718-721 (2016).

15 Vinuesa V, Giménez R, Solano C, Gimeno C, Navarro D. Would kinetic analyses of plasma cytomegalovirus DNA load help to reach consensus criteria for triggering the initiation of preemptive antiviral therapy in transplant recipients? Clin. Infect. Dis. (2016) (In Press).

16 Avetisyan G, Aschan J, Hägglund H, Ringdén O, Ljungman P. Evaluation of intervention strategy based on CMV-specific immune responses after allogeneic SCT. Bone Marrow Transplant. 40 (9), 865-869 (2007)

17 Solano C, Benet I, Remigia MJ et al. Immunological monitoring for guidance of preemptive antiviral therapy for active cytomegalovirus infection in allogeneic stem-cell transplant recipients: a pilot experience. Transplantation 92(4), e17-e20 (2011).

18 Navarro D, Amat P, de la Cámara R et al. Efficacy and safety of a preemptive antiviral therapy strategy based on combined virological and immunological monitoring for active cytomegalovirus infection in allogeneic stem cell transplant recipients. Open Forum Infect. Dis. 3(2), ofw107 (2016). 